\title{
Evaluación de factores organizacionales en la implementación de proyectos integradores de saberes en el segundo nivel de la Carrera de Ingeniería Química
}

\author{
Evaluation of Organizational Factors in the Implementation of Integrating Knowledge \\ Projects in the Second Level of the Chemical Engineering Degree
}

\section{Avaliação de fatores organizacionais na implementação de projetos integradores de saberes no segundo nível da Carreira de Engenharia Química}
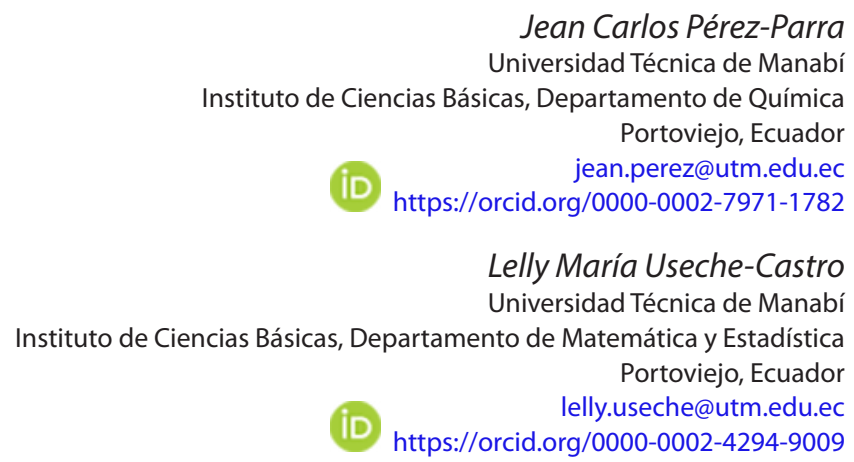

María Antonieta Riera

Universidad Técnica de Manabí

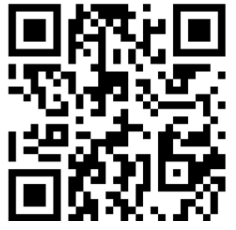

Facultad de Ciencias Matemáticas, Físicas y Químicas, Departamento de Procesos Químicos

Portoviejo, Ecuador

maria.riera@utm.edu.ec

https://orcid.org/0000-0002-7195-2821

Mario Adelfo Batista-Zaldívar

Universidad Técnica de Manabí

Instituto de Ciencias Básicas, Departamento de Química

Portoviejo, Ecuador

mario.batista@utm.edu.ec

https://orcid.org/0000-0002-1623-0332

Julio Cesar Torres-Puentes

Universidad Técnica de Manabí

Instituto de Ciencias Básicas, Departamento de Química

Portoviejo, Ecuador

julio.torres@utm.edu.ec

https://orcid.org/0000-0002-1290-5240

Yulixis Nohemi Cano-De Torres

Universidad Técnica de Manabí

Instituto de Ciencias Básicas, Departamento de Química

Portoviejo, Ecuador

yulixis.cano@utm.edu.ec

https://orcid.org/0000-0003-4442-9039 
http://doi.org/10.15359/ree.25-2.2

http://www.una.ac.cr/educare

educare@una.ac.cr

Recibido • Received • Recebido: 16 / 07 / 2019

Corregido • Revised • Revisado: 19 / 12 / 2020

Aceptado • Accepted • Aprovado: 16 / 02 / 2021

Resumen: En el periodo académico octubre 2017-febrero 2018 se realizaron, por primera vez, proyectos integradores de saberes (PIS) en el segundo nivel de la carrera de Ingeniería Química dela Universidad Técnica de Manabí (UTM). Al finalizar este periodo académico, el $66 \%$ del estudiantado no alcanzó la calificación mínima necesaria para considerar como aprobado los PIS. El objetivo de esta investigación fue evaluar, si durante el periodo académico antes mencionado, fueron considerados los factores organizacionales que se deben tomar en cuenta al implementar estrategias de aprendizaje basadas en la solución de problemas, como los PIS. Para la realización de este estudio se diseñaron dos instrumentos para la recolección de la información, uno para el profesorado y otro para el estudiantado. Entre los aspectos evaluados destacan el número de estudiantes por grupo de trabajo y por curso, criterios para la conformación de grupo, definición de la temática de los PIS, identificación del problema a resolver, acompañamiento mediante tutorías, aprendizaje cooperativo, capacitación del profesorado en PIS, entre otros aspectos. Al comparar la información recabada con otros estudios realizados en esta materia se encontró que varios de los aspectos evaluados en este estudio no han sido establecidos en correspondencia con un aprendizaje mediante PIS. Entre los resultados obtenidos, destaca la necesidad, por parte de la UTM, de contar con un marco normativo para la implementación de los PIS, en las que se adapten los aspectos evaluados a una enseñanza basada en la solución de problemas, en la que se considere la capacitación previa obligatoria tanto del profesorado y como del alumnado en esta estrategia de aprendizaje.

Palabras claves: Proyectos integradores de saberes; ingeniería química; capacitación docente; aprendizaje basado en problemas; trabajo en equipo.

Abstract: In the academic period of October 2017 to February 2018, integrated knowledge projects were carried out for the first time in the second level of the Chemical Engineering career at the Universidad Técnica de Manabí (UTM). At the end of this academic period, $66 \%$ of the students did not reach the minimum qualification necessary to consider the integrating knowledge projects as approved. This research aimed to evaluate if the organizational factors to be taken into account when implementing learning strategies based on problem-solving were considered during the mentioned academic period, such as integrating knowledge projects. This study was conducted by implementing two instruments designed to collect information, one for the teaching staff and the other one for the students. Among the evaluated aspects are the number of students per workgroup and per course, criteria for group formation, the definition of the topic of the integrating knowledge projects, identification of the problem to be solved, support through tutorials, cooperative learning, teachers trained in integrating knowledge projects, among others are highlighted. When the collected information was compared with other studies carried out in this matter, it was found that several of the aspects evaluated in this study were not established in correspondence with learning through integrating knowledge projects. Among the obtained results stands out the need for the UTM to have a normative framework for implementing the integrating knowledge projects in which the evaluated aspects are adapted to teaching based on problem-solving, and prior training is considered mandatory for both teachers and students in this learning strategy.

Keywords: Knowledge integrating projects; chemical engineering; teacher training; problem-based learning; teamwork. 


\begin{abstract}
Resumo: No período letivo de outubro de 2017 a fevereiro de 2018, foram realizados, pela primeira vez, projetos de integração de conhecimento (PIC) no segundo nível da carreira de Engenharia Química da Universidade Técnica de Manabí (UTM). Ao final desse período letivo, $66 \%$ de estudantes não alcançavam a qualificação mínima necessária para considerar aprovado o PIC. O objetivo desta pesquisa foi avaliar, durante o referido período letivo, os fatores organizacionais que devem ser levados em consideração na implementação de estratégias de aprendizagem baseadas na resolução de problemas, como o PIC. Para a realização deste estudo, foram elaborados dois instrumentos de coleta de informações, um para o corpo docente e outro para estudantes. Dentre os aspectos avaliados, destacam-se o número de estudantes por grupo de trabalho e por curso, critérios para formação do grupo, definição do tema do PIC, identificação do problema a ser resolvido, apoio através de tutoriais, aprendizagem cooperativa, formação de professores no PIC, entre outros aspectos. Ao comparar as informações coletadas com outros estudos realizados nesta matéria, constatou-se que vários dos aspectos avaliados neste estudo não foram estabelecidos em correspondência com a aprendizagem pelo PIC. Dentre os resultados obtidos, destaca-se a necessidade do UTM ter um princípio normativo para implantação do PIC, no qual os aspectos avaliados sejam adaptados ao ensino com base na resolução de problemas, na que seja considerada a formação prévia obrigatória de professores e alunos nesta estratégia de aprendizagem.
\end{abstract}

Palavras-chave: Projetos integrativos de conhecimento; engenharia química; formação de professores; aprendizagem baseada em problemas; trabalho em equipe.

\title{
Introducción
}

Las instituciones de educación superior tienen el compromiso con la sociedad de aportar profesionales técnicamente versátiles (multidisciplinariedad), que sean capaces de una comunicación efectiva, de resolver problemas y con responsabilidad social. Esto se logra con la renovación de los currículos educativos que busquen proporcionar entornos de aprendizaje que le permitan al estudiante desarrollar estas características (Savage et al., 2009).

Las universidades, en la búsqueda de alcanzar estos estándares en sus profesionales en formación, han incorporado en sus currículos proyectos integradores de saberes (PIS) como una metodología de aprendizaje colaborativo para el planteamiento y solución de problemas, con un enfoque inter y transdisciplinar (Castillejo-Olán et al., 2017).

El modelo pedagógico tradicional brinda al estudiantado un conocimiento básico, pero no la oportunidad de construir sobre él, como ocurre con los PIS, en los que el aprendizaje pasa de ser un proceso receptivo a uno constructivo, donde la enseñanza se fundamenta en el contacto directo con problemas reales, con lo que genera una estrecha relación entre la práctica y la teoría (Chinowsky et al., 2006), y despierta en el estudiantado un espíritu de investigación e innovación, así como de creatividad para la generación de nuevos conocimientos, pensamientos productivos y motivación para aprender y resolver problemas (de los Ríos et al., 2010).

Los PIS permiten desarrollar, en el estudiantado, habilidades como: trabajo en equipo, comunicación clara y capacidad de equilibrar los impactos sociales, económicos y ambientales de un proyecto (de los Ríos et al., 2010; Savage et al., 2009), también permiten evidenciar logros 
http://doi.org/10.15359/ree.25-2.2

http://www.una.ac.cr/educare

educare@una.ac.cr

de aprendizaje, fortalece el desarrollo de las competencias académicas que se articulan con el perfil de egreso (Moscoso Zamora y Quiñonez Alvarado, 2018).

Ya estudios anteriores han demostrado las ventajas que presenta el aprendizaje mediante PIS en comparación con el método tradicional, pero su implementación no garantiza el éxito. Para lograr óptimos resultados en su ejecución se deben conjugar múltiples factores entre los tres principales actores del proceso de enseñanza (institución, docentes y estudiantes).

La institución juega un papel importante en lo relacionado con dictar e implementar el marco legal, mediante el cual se regirá esta actividad, así como disponer de espacios físicos adecuados donde se puedan desarrollar todas las actividades asociadas a los PIS (bibliotecas, laboratorios, espacios para tutorías, etc.). En el caso del personal docente, juega un papel importante su formación pedagógica en métodos de enseñanza asociados a ambientes reales, así como su disposición a llevar a cabo una actividad con alta interacción docente-estudiante. Y, por último, el estudiantado, que debe contar con hábitos de estudio y conocimientos previos sobre la temática a abordar en los PIS.

La aplicación de los PIS no se debe considerar "como una experiencia única o puntual, sino como un sistema de trabajo continuo a lo largo de los estudios" (Egido et al., 2007, p. 96), en los que se planifica la temática a desarrollar en cada semestre, cuyo nivel de complejidad deberá implementarse en forma secuencial y progresiva. Cada asignatura se integrará aportando a la construcción del proyecto (Moscoso Zamora y Quiñonez Alvarado, 2018).

En 2016, la Universidad Técnica de Manabí (UTM) de Ecuador aprobó el rediseño curricular de todas sus carreras. Con este rediseño se incorporaron los PIS en cada uno de los niveles de todas las carreras de la UTM. En el caso particular de Ingeniería Química, los PIS se implementaron por primera vez en el segundo nivel de la carrera en el período académico octubre 2017-febrero 2018.

Un aspecto a resaltar luego de la primera implementación de los PIS en el segundo nivel de la carrera de Ingeniería Química fue que el $66 \%$ del estudiantado no obtuvo la nota mínima necesaria para aprobar el proyecto. Con esta investigación se persigue evaluar, como una posible causa del bajo rendimiento obtenido por el estudiantado, si durante el periodo académico en estudio, factores de índole organizacional estuvieron en correspondencia con una estrategia de aprendizaje basada en la solución de problemas en grupo, como lo son los PIS.

\section{Organización de los PIS en la UTM}

\section{Aspectos fundamentales de los PIS}

La organización los PIS en la UTM se llevó tal como lo establece Hewitt Ramírez (2007) en su trabajo: 
http://doi.org/10.15359/ree.25-2.2

La elaboración del proyecto integrador implica que los estudiantes al cursar cada uno de sus semestres académicos delimiten una problemática y visualicen su solución con los recursos científicos disponibles. La integración del conocimiento se logra a partir del diseño y ejecución de un ejercicio de investigación que se articula con la intervención en el contexto en el que participan. (p. 238)

Los PIS contemplan varias fases o etapas. El proyecto integrador considerado en este estudio se desarrolló en dos etapas de ocho semanas cada una, para un total de 16 semanas, que es la duración de un periodo académico en la UTM. En la Tabla 1 se presentan las actividades realizadas por docentes y estudiantes en cada etapa del proyecto. La primera etapa consistió en la presentación de una propuesta de investigación enmarcada en la temática central del proyecto integrador. En la segunda etapa el estudiantado desarrolló la investigación propuesta, integrando los contenidos de las asignaturas que forman parte del proyecto. Al final del periodo académico se presentaron los resultados obtenidos en los PIS, tanto de forma oral como escrita, para su correspondiente evaluación.

Tabla 1: Etapas, fases y actividades de los PIS.

\begin{tabular}{|c|c|c|c|}
\hline Etapa & Fase & Actividades del personal docente & Actividades del estudiantado \\
\hline \multirow{4}{*}{$\begin{array}{l}\frac{\pi}{0} \\
\frac{0}{0} \\
\frac{0}{0} \\
\frac{0}{0} \\
\stackrel{\frac{0}{2}}{2}\end{array}$} & Organización & $\begin{array}{l}\text { - Unifica criterios con otras personas docentes } \\
\text { de las asignaturas del PIS sobre cronograma, } \\
\text { conformación de grupos, momentos, } \\
\text { técnicas e instrumentos de evaluación. }\end{array}$ & $\begin{array}{l}\text { - Inscribe materias del proyecto } \\
\text { integrador. }\end{array}$ \\
\hline & Activación & $\begin{array}{l}\text { - Presenta el proyecto integrador en la } \\
\text { primera clase del período académico. }\end{array}$ & $\begin{array}{l}\text { - Forman equipos de trabajo. } \\
\text { - Activación del conocimiento. }\end{array}$ \\
\hline & $\begin{array}{l}\text { Investigación para } \\
\text { propuesta del } \\
\text { proyecto }\end{array}$ & $\begin{array}{l}\text { - Sigue los avances en la selección del tema, } \\
\text { elaboración de la propuesta y el plan de } \\
\text { trabajo, a través de tutorías. } \\
\text { - Hace un seguimiento a los recursos utilizados } \\
\text { por el estudiantado en el proyecto, haciendo } \\
\text { recomendaciones de ser el caso. }\end{array}$ & $\begin{array}{l}\text { - Utiliza puntos clave para orientar } \\
\text { su búsqueda de información. } \\
\text { - Organiza la información. }\end{array}$ \\
\hline & $\begin{array}{l}\text { Evaluación de la } \\
\text { propuesta del } \\
\text { proyecto }\end{array}$ & $\begin{array}{l}\text { - Propicia la discusión sobre la investigación } \\
\text { entre estudiantes de un mismo grupo con } \\
\text { miras a lograr en estos la reflexión conjunta. } \\
\text { - Mide el desarrollo de competencias. }\end{array}$ & $\begin{array}{l}\text { - Entrega y discute con su docente } \\
\text { de tutoría la propuesta del } \\
\text { proyecto. } \\
\text { - Evalúa la actividad. }\end{array}$ \\
\hline \multirow{2}{*}{ 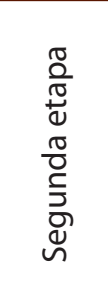 } & Resolución & $\begin{array}{l}\text { - Conduce al estudiantado en la búsqueda } \\
\text { de información y en el planteamiento de } \\
\text { soluciones a la propuesta planteada. }\end{array}$ & $\begin{array}{l}\text { - Discute y busca información con } \\
\text { base en la retroalimentación. } \\
\text { - Diseña soluciones. } \\
\text { - Realiza el informe final. }\end{array}$ \\
\hline & $\begin{array}{l}\text { Evaluación del } \\
\text { proyecto final }\end{array}$ & $\begin{array}{l}\text { - Dirige discusión y reflexión grupal. } \\
\text { - Evalúa el desarrollo de habilidades y } \\
\text { competencias. }\end{array}$ & $\begin{array}{l}\text { - Presenta soluciones al grupo y } \\
\text { discute su pertinencia. } \\
\text { - Evalúa la actividad. }\end{array}$ \\
\hline
\end{tabular}

Nota: Adaptado de Villalobos Delgado et al. (2016). 
http://doi.org/10.15359/ree.25-2.2

http://www.una.ac.cr/educare

educare@una.ac.cr

Los PIS en la UTM se organizan en cada nivel a partir de la integración de tres asignaturas, donde la cátedra integradora será aquella que más contribuya al perfil de egreso. En el caso del segundo nivel de la carrera de Ingeniería Química, las tres asignaturas que conforman el proyecto integrador son: Química Orgánica y Laboratorio I (cátedra integradora), Introducción a la Ingeniería Química e Introducción a la Investigación Científica. La Tabla 2 contiene tanto los contenidos mínimos como los logros de aprendizaje de las asignaturas que conforman el proyecto integrador del segundo nivel de la carrera de Ingeniería Química de la UTM.

Tabla 2: Contenidos mínimos y logros de aprendizaje de las asignaturas que forman parte del proyecto integrador del segundo nivel de la carrera de Ingeniería Química de la UTM

\begin{tabular}{|c|c|c|}
\hline Asignatura & Contenidos mínimos & Logros de aprendizaje \\
\hline $\begin{array}{l}\text { Química Orgánica y } \\
\text { Laboratorio I (asignatura } \\
\text { integradora) }\end{array}$ & $\begin{array}{l}\text { - Estructura del carbono y enlaces } \\
\text { (elementos biogenésicos). } \\
\text { - Nomenclatura de hidrocarburos } \\
\text { saturados e insaturados, isomería } \\
\text { estructural, grupos funcionales. }\end{array}$ & $\begin{array}{l}\text { - Aplicar los conocimientos de la química } \\
\text { orgánica para diferenciar el comportamiento } \\
\text { y la estructura de los compuestos orgánicos. }\end{array}$ \\
\hline $\begin{array}{l}\text { Introducción a la } \\
\text { Ingeniería Química }\end{array}$ & $\begin{array}{l}\text { - Unidades y dimensiones. } \\
\text { - Variable de procesos. } \\
\text { - Estequiometria. }\end{array}$ & $\begin{array}{l}\text { - Identificar los diversos sistemas con sus } \\
\text { unidades y dimensiones para realizar } \\
\text { transformaciones. } \\
\text { - Examinar las diferentes variables de procesos } \\
\text { mayormente aplicadas a la Ingeniería } \\
\text { Química para resolución de problemas. } \\
\text { - Aplicar fundamentos matemáticos, físicos, } \\
\text { químicos y biológicos para modelar y resolver } \\
\text { problemas de relaciones de materia en } \\
\text { Ingeniería Química. }\end{array}$ \\
\hline $\begin{array}{l}\text { Introducción a la } \\
\text { Investigación Científica }\end{array}$ & $\begin{array}{l}\text { - Investigación científica. Conceptos } \\
\text { fundamentales. } \\
\text { - El método científico. } \\
\text { - Práctica de la ciencia. }\end{array}$ & $\begin{array}{l}\text { - “Reconocer los rasgos clave de la } \\
\text { investigación científica. ... } \\
\text { - Reflexionar sobre las implicaciones sociales } \\
\text { [y ambientales] de los avances científicos y } \\
\text { tecnológicos. } \\
\text { - Reconocer cuestiones susceptibles de ser } \\
\text { investigadas científicamente. } \\
\text { - Identificar términos clave para la búsqueda } \\
\text { de información científica." (Organización para } \\
\text { la Cooperación y el Desarrollo Económico } \\
\text { [OCDE], 2007, p. 30). } \\
\text { - Elaborar ensayo bibliográfico sobre un tema } \\
\text { susceptible de ser investigado. } \\
\text { - Identificar las principales etapas del método } \\
\text { científico desde el punto de vista práctico. }\end{array}$ \\
\hline
\end{tabular}

Nota: Información obtenida, con autorización, del documento final aprobado del rediseño de la carrera de Ingeniería Química de la Universidad Técnica de Manabí (Universidad Técnica de Manabí [UTM], 2016). 
http://doi.org/10.15359/ree.25-2.2

En cada nivel de cada carrera se estableció un tema central para ser abordado a través de los proyectos integradores. Para el segundo nivel de la carrera de Ingeniería Química se fijó como temática central de los PIS, establecer la carga contaminante resultante de los procesos industriales, y abordar dicho estudio mediante el método científico (UTM, 2016).

En la UTM se ha planteado que las actividades del proyecto integrador sean incorporadas al programa académico de cada una de las asignaturas que forman parte del proyecto, manteniendo a su vez los mismos contenidos mínimos de las asignaturas, antes del rediseño de 2016.

Para el momento de este estudio, 181 estudiantes cursaban el segundo nivel de la carrera de Ingeniería Química. Estos se distribuyeron entre los varios cursos ofertados para cada materia (Tabla 3). Un total de nueve profesores impartieron los 14 cursos de las tres asignaturas del proyecto integrador.

Tabla 3: Relación de docentes y ambientes en cada una de las materias del proyecto integrador

\begin{tabular}{lcc}
\hline \multicolumn{1}{c}{ Asignatura } & Cursos & Profesores \\
\hline Química Orgánica y Laboratorio I (asignatura integradora) & 4 & 2 \\
Introducción a la Ingeniería Química & 5 & 3 \\
Introducción a la Investigación Científica & 5 & 4 \\
\hline Total & 14 & 9 \\
\hline
\end{tabular}

Nota: Elaboración propia.

\section{- Organización de los grupos de trabajo}

En la fase de organización se acordó que el estudiantado, desde los cursos de la asignatura integradora, se conformarían en grupos de máximo seis participantes para la elaboración del proyecto. Se esperaba que los 181 estudiantes cursantes del segundo nivel se conformaran en al menos 30 grupos. Se le recomendó al estudiantado que utilizara como criterio para seleccionar a los compañeros y las compañeras de grupo, aquel estudiantado que perteneciera a los mismos cursos en las tres asignaturas, con la finalidad de tener similar distribución horaria de las clases, lo que facilitaría la asistencia a tutorías, esto también permitiría que hubiese coincidencia en las horas libres y con ello se pudieran realizar las actividades asociadas al proyecto.

En el proceso de conformación de los grupos, parte del estudiantado se organizó rápidamente siguiendo el criterio establecido. El estudiantado que tardó más en organizarse tuvo dificultad para encontrar pares que compartieran los cursos de las otras materias del proyecto, lo que originó que existieran grupos donde el estudiantado solo coincidía en el curso de la asignatura integradora. 
http://doi.org/10.15359/ree.25-2.2

http://www.una.ac.cr/educare

educare@una.ac.cr

Aun cuando solo 167 de los 181 estudiantes inscritos en la asignatura integradora presentaron propuesta de proyecto, finalmente quedaron organizados en 33 grupos, de los cuales solo el $42,42 \%$ estuvo formado por seis estudiantes, y el resto se conformó con un número menor de estudiantes. En la Tabla 4 se observa la distribución de grupos por número de integrantes.

Tabla 4: Distribución de los grupos por número de integrantes

\begin{tabular}{cccc}
\hline Integrantes por grupo & Grupos & Total de estudiantes & Porcentaje de grupos (\%) \\
\hline 2 & 1 & 2 & 3,04 \\
3 & 2 & 6 & 6,06 \\
4 & 5 & 20 & 15,15 \\
5 & 11 & 55 & 33,33 \\
6 & 14 & 84 & 42,42 \\
\hline TOTAL & 33 & 167 & 100,00 \\
\hline
\end{tabular}

Nota: Elaboración propia.

Luego de las indicaciones dadas para la conformación de los grupos de trabajo, solo cuatro de los 33 grupos (12\%) se conformaron por estudiantes que compartían los mismos cursos en las tres materias, el resto de los grupos tuvo niveles de coincidencia en los cursos variada. En la Figura 1 se muestran, mediante un esquema, los cursos ofertados para cada una de las materias que formaban parte del proyecto integrador durante el periodo académico en estudio. Los cursos se muestran con su respectiva codificación y con el número de estudiantes inscritos entre paréntesis. En la parte inferior de la Figura 1 aparece el total de estudiantes por materia, y se representa como están distribuidos los estudiantes de cuatro grupos con diferentes niveles de coincidencia de sus integrantes entre los cursos de las tres materias del proyecto. El grupo signado con el número 14 (representado con puntos azules) fue uno de los cuatro cursos con $100 \%$ de coincidencia de sus participantes en los mismos cursos de las tres materias. En el caso del grupo 17 (representado con puntos de color naranja), el estudiantado solo coincidía en el curso de Química Orgánica y Laboratorio I, estaban en tres cursos de Introducción a la Ingeniería Química ( $A, C$ y D) y en tres cursos de Introducción a la Investigación Científica ( $T$, $U$ y W). La mayoría de los grupos tuvo una distribución similar al grupo 17. En la Figura 1 también se representa la distribución del estudiantado de otros grupos (grupo 32 y grupo 9) con menor nivel de coincidencia de sus estudiantes en los cursos de las tres asignaturas.

Los puntos grises y verdes con borde negro, en la Figura 1, representan a estudiantes que solo cursaron Química Orgánica y Laboratorio I y que fueron incorporados a los grupos de trabajo de los PIS. 
http://doi.org/10.15359/ree.25-2.2

http://www.una.ac.cr/educare

Figura 1: Distribución de estudiantes pertenecientes a los grupos de los PIS entre los diferentes cursos de las asignaturas integradoras

\begin{tabular}{|c|c|c|}
\hline $\begin{array}{c}\text { Cursos de Química Orgánica y } \\
\text { Laboratorio I }\end{array}$ & $\begin{array}{c}\text { Cursos de Introducción a la } \\
\text { Ingeniería Química }\end{array}$ & $\begin{array}{c}\text { Cursos de Introducción a la } \\
\text { Investigación Científica }\end{array}$ \\
\hline $\mathrm{A}(50)$ & & $\mathrm{S}(33)$ \\
\hline & & \\
\hline $\mathrm{C}(41)$ & & \\
\hline $\mathrm{B}(49)$ & & \\
\hline
\end{tabular}

Grupo 17 (๑); grupo 14(๑); grupo 32 (๑) y grupo 9 (๑).

Nota: Elaboración propia.

\section{- Acompañamiento y evaluación de los PIS}

Los PIS fueron concebidos en la UTM de tal manera que cada docente de cada una de de las tres asignaturas brindó acompañamiento académico al estudiantado durante la realización del proyecto, y al final de cada etapa realizaran la evaluación. Pero, si cada docente que imparte clases al estudiantado de un grupo con una distribución entre los cursos de las tres materias, como la del grupo 17 (Figura 1), debe intervenir en el acompañamiento y evaluación del 
http://doi.org/10.15359/ree.25-2.2

http://www.una.ac.cr/educare

educare@una.ac.cr

proyecto integrador de dicho grupo, esta tarea se convertiría en un verdadero desafío, debido las diferencias de criterio que pudieran existir entre un grupo de 7 docentes que debieran involucrarse en el acompañamiento y evaluación de los PIS. Esta situación llevó a tomar la decisión de designar a cada grupo de trabajo, entre el profesorado de las materias del proyecto integrador, un profesor o profesora de tutoría para que se encargase tanto de asesorar al estudiantado durante la realización del proyecto, así como de evaluar el PIS en todas sus etapas.

\section{- Presentación de la propuesta y distribución de grupos de trabajo}

Según la planificación planteada para los PIS, el estudiantado debía presentar una propuesta de proyecto en la semana seis del período académico. Posterior a esta presentación se realizó la designación del personal tutor entre los nueve docentes que imparten las asignaturas del proyecto, teniendo como criterio designar en la tutoría de un grupo a aquel profesorado que tuviese más estudiantes en dicho grupo; tratando, en la medida de lo posible, de que a quienes atienden más cursos de las materias del proyecto integrador se les asignara un mayor número de grupos de trabajo.

La distribución de cursos y grupos de los proyectos por docente se muestran en la Figura 2. En ella se constata que la mayoría del profesorado (seis de nueve, el 66,7\%), tuvo solo un curso de las asignaturas del proyecto integrador (Figura 2a). En la distribución de los grupos se observa que la mayoría del profesorado (cinco de nueve, el 55,6\%), tuvo a cargo tres grupos de trabajo (Figura 2b).

Figura 2: (a) Relación de cursos por profesor

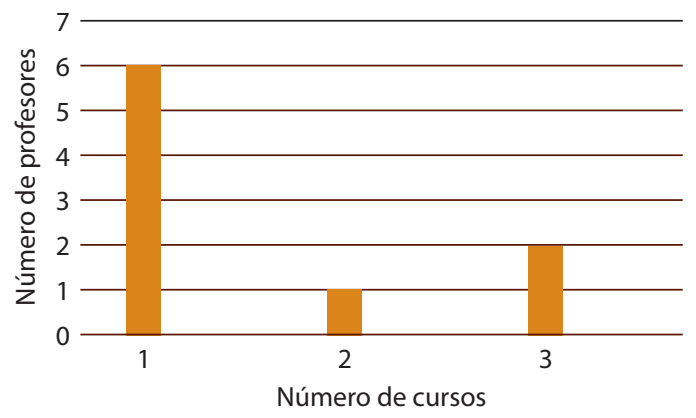

(a) (b) Relación de grupos del PIS por profesor

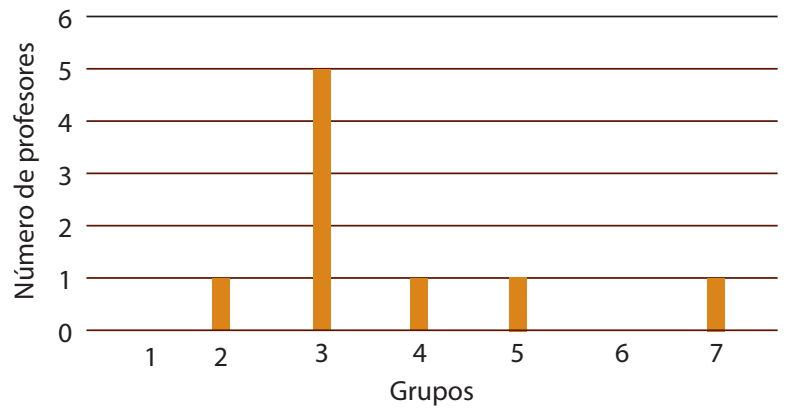

(b)

Nota: Elaboración propia. 
http://doi.org/10.15359/ree.25-2.2

\section{- Evaluación de los PIS y ponderación de la calificación}

Los PIS se evaluaron en cada una de las dos etapas. En la primera etapa del PIS durante la semana 8 del periodo académico, se evaluó la presentación escrita de la propuesta del proyecto realizada por cada grupo. En la segunda etapa se evaluaron los resultados de la investigación realizada a partir del proyecto integrador, la cual fue presentada tanto escrita como oralmente. Esta evaluación se realizó durante la semana 16.

EnlaUTMseha establecido que la calificación de las asignaturas esté entreceroy 100 puntos, se exige para la aprobación una calificación mínima de 70 puntos. Los periodos académicos se dividen en dos ciclos de ocho semanas cada uno, durante los cuales el estudiantado puede acumular un máximo de 50 puntos en cada ciclo, entre los diferentes componentes de evaluación establecidos por la institución: docencia (15 puntos), autónomo (10 puntos), experimental (10 puntos) y un examen que se realiza al final de cada ciclo (15 puntos).

El proyecto integrador se incluyó dentro del componente de docencia y se evalúa entre cero y 10 puntos en cada ciclo, permite que el estudiantado acumule hasta 20 puntos en el periodo académico, lo que representa el $20 \%$ de la calificación máxima de cada asignatura.

\section{Materiales y métodos}

Con todos los aspectos organizacionales e institucionales mencionados anteriormente se implementaron los PIS en el segundo nivel de la carrera de Ingeniería Química de la UTM durante el periodo académico octubre 2017-febrero 2018. Luego de la implementación de esta experiencia de aprendizaje, se puede resaltar, entre otros aspectos, que el $66 \%$ del estudiantado no alcanzó la calificación mínima para considerar como aprobada dicha actividad (14 puntos).

En este estudio se plantea una investigación de campo, cualitativa-cuantitativa, no experimental, con la que se busca determinar, si los factores de índole organizacional, establecidos durante el periodo en estudio, correspondían con una estrategia de aprendizaje basada en la solución de problemas en grupo.

Para la recolección de la información se construyeron dos instrumentos, uno para el estudiantado y otro para el profesorado, con preguntas abiertas y cerradas.

Al estudiantado se le aplicó una encuesta con 20 preguntas, relacionadas con la conformación de los grupos, dificultad en el planteamiento de la propuesta del proyecto, presencia de estudiantes en funciones de líder y estudiantes que no participaron en las actividades del proyecto, dificultad en la integración del estudiantado a los grupos, número de estudiantes por grupo, conformidad con la calificación obtenida, asistencia a tutorías, entre otros aspectos. De 167 estudiantes que presentaron una propuesta para el PIS al inicio del 
http://doi.org/10.15359/ree.25-2.2

http://www.una.ac.cr/educare

educare@una.ac.cr

periodo académico, 143 completaron la actividad mediante la presentación de los resultados de la investigación realizada a través del PIS al final del periodo académico. El instrumento para la recolección de la información fue respondido por 123 de 143 estudiantes que completaron las dos etapas del PIS, es decir, el $86 \%$ del estudiantado.

La encuesta desarrollada y aplicada al profesorado contó con 22 preguntas que abordaron aspectos como formación profesional, experiencia en docencia universitaria y en estrategias de aprendizaje basada en problemas. En lo que respecta a la implementación de los PIS, se les consultó sobre el tiempo de dedicación a asignaturas integradoras en comparación con el tiempo de dedicación a otras asignaturas que no pertenecen a proyectos integradores, su apreciación sobre el número de estudiantes por grupo establecido y sobre la temática central de los PIS. También se les consultó sobre la frecuencia con la que el estudiantado asistía a tutorías, entre otros aspectos. La totalidad de nueve docentes que impartieron los diferentes cursos de las tres materias integradoras respondieron la encuesta de este estudio.

Ambos cuestionarios fueron validados por tres expertos en pedagogía y química. Para una mejor facilidad en el tratamiento y análisis de los datos, y debido al número de personas a encuestar, el cuestionario aplicado al estudiantado fue adaptado al formato de formularios de google y fue enviado por correo al estudiantado para que lo completara.

\section{Resultados y discusión}

\section{En relación con el estudiantado}

\section{- Conformación de grupos de trabajo}

En el segundo nivel de la carrera de Ingeniería Química para el periodo académico en estudio se esperaría que el estudiantado curse en paralelo las tres asignaturas del PIS (UTM, 2016), pero esto depende de muchos factores, entre los que destaca la aprobación de las materias que son prerrequisito de las asignaturas integradoras. En la Figura 3 se muestra la relación de las asignaturas cursadas por el estudiantado para el periodo en estudio. El 80,5\% del estudiantado cursó las tres asignaturas del proyecto integrador. Del 19,5\% restante, hubo estudiantes que no cursaron Introducción a la Investigación Científica, lo que representa una dificultad en la implementación del proyecto integrador para este estudiantado, pues en esta asignatura se aprenden los conocimientos necesarios para llevar a cabo investigaciones de carácter científico, como la que se desarrollaría en los PIS. 
http://doi.org/10.15359/ree.25-2.2

http://www.una.ac.cr/educare educare@una.ac.cr

Figura 3: Relación de materias cursadas por el estudiantado

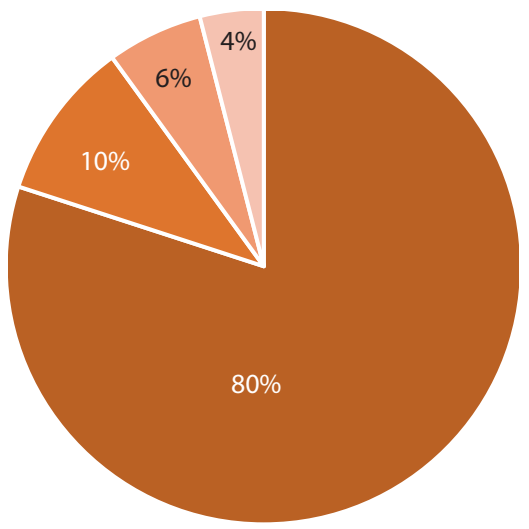

Estudiantes que cursaron las tres asignaturas del proyecto integrador

Estudiantes que solo cursaron Química Orgánica y Laboratorio

Estudiantes que cursaron Química Orgánica y Laboratorio e Introducción a la Investigación Científica

Estudiantes que cursaron Química Orgánica y Laboratorio e Introducción a la Ingeniería Química

Nota: Elaboración propia.

En relación con la conformación de los grupos de trabajo, aun cuando se le indicó al estudiantado que el criterio para seleccionar integrantes de los grupos fuese que compartieran los mismos cursos en las asignaturas del proyecto; el 44,7\% expresó haber tenido algún tipo de dificultad para cumplir este criterio, entre las que destacan: desconocimiento en parte del profesorado del criterio de organización establecido, no conocer al resto del estudiantado, y finalmente, dificultad para unirse a algún grupo ya conformado luego de haber transcurrido las primeras semanas del periodo académico. Otros estudios han indicado que, en cuanto a la conformación de grupos, se debe tomar en cuenta el perfil del estudiantado, aun cuando esto representa un verdadero desafío, debido a la diferencia existente entre los perfiles del estudiantado. Entre los aspectos más importantes a considerar, en este sentido, están el nivel de conocimiento previo y el interés por la asignatura, este último puede originar dificultades, y, por ende, retrasos en la realización del proyecto (Fioravanti et al., 2018), llegan a imposibilitar, en algunos casos, su finalización. Se hace necesario, entonces, el establecimiento de un mecanismo efectivo mediante el cual se organicen los grupos de trabajo para los PIS, basado en los criterios que mejor se adapten al estudiantado de la UTM, con el que se superen las dificultades expuestas anteriormente, y que este, a su vez, se realice previo a la iniciación del periodo académico. 
http://doi.org/10.15359/ree.25-2.2

http://www.una.ac.cr/educare

educare@una.ac.cr

\section{- Integración del estudiantado a las actividades del PIS}

Mediante el instrumento aplicado para la recolección de la información se quiso conocer si se presentaron dificultades por parte del estudiantado para integrase a las actividades del proyecto integrador. Sobre este aspecto, el 34,1\% del estudiantado expresó haber tenido dificultades para integrarse a las actividades que se debían realizar, debido, entre otros aspectos, al poco tiempo disponible para ejecutar dichas actividades, ya que otras actividades académicas (tareas, test, informes, etc.) que se asignan tanto desde las materias integradoras como desde aquellas que no lo son, demandan la mayor parte del tiempo. Además, la diferencia entre los horarios de clases del estudiantado impide poder reunirse para realizar las actividades del proyecto fuera de la jornada académica. A esto se suma la imposibilidad de que puedan reunirse en días libres, debido a lo distante que se encuentran sus lugares de residencia. Se debe, entonces, considerar también la disposición real de tiempo por parte del estudiantado para atender las actividades del proyecto integrador, frente al resto de actividades académicas con las que debe cumplir en un mismo periodo académico.

\section{- Planteamiento de la propuesta}

En la fase del planteamiento del problema, el $82,1 \%$ del estudiantado indicó tener dificultad para reconocer algún problema del entorno al que se le pudiera dar solución a través del proyecto integrador. En este aspecto, coincide el estudiantado con el profesorado en que la principal dificultad estuvo en que el proyecto integrador debía estar enmarcado, tanto por los temas a abordar en las tres asignaturas que conforman el proyecto integrador, como por la temática central establecida en el rediseño de la carrera. Por tratarse de un conocimiento aún no adquirido por el estudiantado, fue difícil que pudieran plantear un proyecto dentro de este marco establecido. La respuesta más representativa a este aspecto, dada por el estudiantado fue: No sabíamos cómo empezar o qué se debía hacer (EST37).

Lo anterior está en concordancia con lo planteado por Egido et al. (2007), quienes indican que, en la etapa inicial de la implementación de métodos basados en solución de problemas, el estudiantado puede presentar "falta de conocimientos previos y de hábitos de trabajo autónomo, por lo que no es extraño que se produzca un cierto desconcierto y afloren los sentimientos de inseguridad ante la actividad" (p. 96), así como presión o estrés. De hecho, se ha determinado que la aplicación de este método de aprendizaje es más útil en niveles superiores de la carrera que en los iniciales, debido precisamente a la necesidad de conocimientos previos por parte del estudiantado en las áreas sobre las que se realizarían los PIS (Egido et al., 2007). Por ello representa un desafío para el estudiantado la realización de una investigación científica de carácter aplicado, en la que se requiera un conocimiento que aún no ha adquirido. En este sentido, el estudiantado preferiría que el problema a abordar en el proyecto integrador lo asigne el personal tutor y no que sea el estudiantado quien deba buscarlo del entorno. Con 
http://doi.org/10.15359/ree.25-2.2

esto también se evitaría que haya proyectos en los que se aborden los mismos problemas. Una propuesta que permitiría superar estas dificultades por parte del estudiantado podría ser la creación de un banco de problemas por parte del profesorado que pudieran ser resueltos mediante la realización de PIS.

\section{- Aspecto cooperativo de los PIS}

Los PIS requieren de un mayor grado de responsabilidad por de sus estudiantes, especialmente en lo que respecta a su propio proceso de aprendizaje (de los Ríos et al., 2010). En este sentido, todo el estudiantado debe tener una participación activa en la realización del proyecto. En este estudio, el 56,1\% del estudiantado expresó haber tenido en sus respectivos grupos integrantes que no contribuyeron en la realización del proyecto, lo cual deja en evidencia la poca disposición de una parte a trabajar en el proyecto, faltando con ello a la responsabilidad individual con la que cada estudiante debe responder tanto a su propio aprendizaje como al de las demás personas (Johnson et al., 1999). Al inicio del periodo académico, no solo se debe describir al estudiantado las diferentes actividades que se deben realizar en los PIS, sino también se debe resaltar la importancia del trabajo cooperativo de este tipo de estrategias de aprendizaje, así como la posibilidad que tendrá, mediante la participación activa en las diferentes actividades del proyecto, de desarrollar competencias personales y profesionales, mejorar estrategias de comunicación y sus habilidades interpersonales, además de favorecer sus capacidades de planificación, organización, análisis, síntesis y razonamiento crítico de la información (Lanza Escobedo y Barrios Fernández, 2012). Otras personas autoras han expuesto, sobre este aspecto, que previo a la aplicación de esta metodología, se debe formar tanto al profesorado como al estudiantado en metodologías de enseñanzas basadas en equipos de trabajo, esto con la finalidad de desarrollar tanto actitudes como habilidades necesarias cuando se trabaja en grupo (Egido et al., 2007).

En la presentación oral que debía realizarse al final del periodo académico, en la que se presentaban los resultados de la investigación, debía participar la totalidad de integrantes de cada grupo. Algún estudiantado, para evitar ser perjudicado con una baja calificación en la evaluación final del proyecto, decidió, en consenso, excluir al estudiantado que no había participado en las actividades del proyecto, por no contar con los conocimientos necesarios para la defensa oral. Esto fue motivo, en parte, de que en solo 18 de los 33 grupos (el 54,54\%) se mantuvieran completos hasta la presentación final del proyecto.

Cuando se trabaja en grupo también se presentan situaciones en las que algún estudiantado asume el rol de líder dentro de los grupos, lo que contraviene con el principio del aprendizaje cooperativo en el cual se debe evitar el surgimiento de actitudes pasivas, como la mencionada anteriormente, pero también se debe evitar el surgimiento en algún estudiantado de actitudes dominantes. Sobre este aspecto se le consultó al estudiantado si existió en su grupo una persona 
http://doi.org/10.15359/ree.25-2.2

http://www.una.ac.cr/educare

educare@una.ac.cr

o varias que ejercieran funciones de líder dentro del grupo, asignando las actividades a desarrollar al resto de las personas participantes, a lo que el 47,2\% indicó que sí hubo estudiantes con estas características en su grupo. Esto también se hizo evidente en respuestas como:

Yo era la persona que tenía que estar más al pendiente del Proyecto y asignar tareas a los integrantes que casi no cumplían. (EST101)

\section{Era yo quien organizaba y me encargaba de casi todo el proyecto. (EST12)}

Se puede inferir, a partir de las respuestas dadas por el estudiantado, que estas actitudes dominantes en algunos integrantes de los grupos podrían surgir como respuesta natural a la actitud pasiva que adopta otro estudiantado. Esto hace necesario establecer mecanismos que permitan hacer seguimiento y evaluación de la participación individual del estudiantado en las diferentes actividades del proyecto.

\section{- Número de integrantes por grupo}

En relación con el número de estudiantes por grupo, el cual se había establecido en máximo seis estudiantes, el 36,6\% del estudiantado manifestó estar de acuerdo con este número máximo de participantes por grupos, mientras que la mayoría expresó que el número de integrantes debería ser menor, debido, principalmente, a que grupos con seis integrantes favorecen al estudiantado que no cumple con las tareas asignadas, conocido como estudiantes polizón (aquel estudiante que rehúye de su responsabilidad), y que deja las tareas y responsabilidades del proyecto solo en algunos o algunas de sus integrantes, tal como se ha detectado en otros estudios, en los que"no todos los estudiantes aportaban de la misma manera a la resolución de problemas, y, en general, solo uno o dos de los miembros de cada grupo eran los que llevaban adelante la mayor parte de la tarea" (Fernández y Aguado, 2017, p. 157), de hecho, algunas personas autoras aconsejan que los grupos no sean mayores a cinco integrantes (Moscoso Zamora y Quiñonez Alvarado, 2018). El efecto polizón se puede evitar asegurando la aportación de todo el estudiantado a través de la división de tareas, roles y evaluaciones (Lanza Escobedo y Barrios Fernández, 2012).

\section{- Trabajar en PIS de niveles superiores formando grupo con el mismo estudiantado}

Con la finalidad de conocer el grado de satisfacción del estudiantado con respecto al trabajo realizado por sus compañeros de grupo, se consultó al estudiantado si realizaría el proyecto integrador de niveles superiores con el mismo grupo. El 34\% indicó que sí lo haría, otro 39\% preferiría trabajar con solo algunos de sus compañeros o compañeras, mientras que el $17,1 \%$ preferiría hacerlo con otros u otras estudiantes y el 9,8\% restante elegiría realizarlo sin compañía. Esto último es común en aquel estudiantado que ejerce función de líder en el grupo, de allí la percepción de que él o ella, sin ayuda, podría realizar el proyecto. 


\section{- Asistencia a tutorías}

En cuanto a la asistencia del estudiantado a tutorías, aspecto importante que permite el acompañamiento por parte del profesorado en la realización del proyecto; el 17,9\% del estudiantado expresó no haber asistido a tutoría debido principalmente a que, en el horario establecido por el profesorado para esta actividad, el estudiantado debe atender clases de otras asignaturas. Además, el 57,7\% del estudiantado planteó la imposibilidad que todas las personas integrantes del grupo asistieran juntos a tutoría, debido a la diferencia en el horario de clases, situación que también impide que el estudiantado pudiera reunirse durante la semana. Otra situación recurrente expuesta por el estudiantado fue la falta de comunicación entre las personas integrantes del grupo, que afectó no solo la asistencia a tutorías, sino también la realización de otras actividades asociadas al proyecto. Se hace necesario establecer un horario para tutorías por parte del profesorado, y que dicho horario no coincida con el horario en que el estudiantado deba asistir a otras asignaturas.

\section{- Satisfacción con la calificación obtenida en el PIS}

Debido a que fueron nueve docentes quienes evaluaron a los 33 grupos que se constituyeron para la realización de los PIS, se quiso conocer la percepción del estudiantado en relación con la calificación asignada al proyecto presentado para cada grupo por el personal tutor. Sobre este aspecto, el 30,1\% del estudiantado indicó no estar satisfecho con la calificación recibida, manifestando que de haber sido otro el personal docente que hubiese evaluado el proyecto, la calificación obtenida hubiese sido mejor. Esta situación genera en el estudiantado la percepción que las calificaciones obtenidas dependían del personal tutor asignado, situación que evidencia la necesidad de establecer rúbricas de evaluación, de tal manera que la calificación asignada sea la misma, independientemente del profesorado que realice la evaluación.

Algún estudiantado ha expuesto que el nivel de exigencia por parte de algún profesorado sobre las actividades a desarrollar en los PIS no se corresponde, ni con el tiempo ni con los materiales con los que se dispone para la realización del proyecto. Esta situación también podría solucionarse con la implementación de rúbricas de evaluación.

\section{- Designación de tutores a grupos}

Una vez presentada todas las propuestas de los PIS, se procedió con la designación de personal tutor, para que este, a su vez, realizara la evaluación a dichas propuestas. El estudiantado durante la realización de la propuesta realizó consultas y (o) recibió tutoría de parte de algún profesorado que impartía las materias de los PIS, mientras que la evaluación de la propuesta la realizó el sujeto tutor designado. En este sentido, el estudiantado indicó que la designación de los tutores o tutoras debió llevarse a cabo desde el inicio del periodo académico, y con ello poder contar con su acompañamiento desde el inicio de la realización de la propuesta, 
http://doi.org/10.15359/ree.25-2.2

http://www.una.ac.cr/educare

educare@una.ac.cr

lo que hubiese permitido obtener una mejor calificación en esta primera etapa del PIS. Para verificar, si el planteamiento realizado por el estudiantado se ajustaba a la realidad, se realizó una comparación de los promedios de las calificaciones obtenidas en la propuesta del proyecto con las obtenidas en la presentación final, ya que en esta última etapa sí se había contado con el acompañamiento tutorial. Los resultados de esta comparación se muestran en la Figura 4 $y$, contrario a lo expuesto por el alumnado, el promedio de las calificaciones obtenidas en la propuesta $(6,2$ puntos) fue mayor al promedio de las calificaciones obtenidas en la presentación final del proyecto (5,61 puntos).

Figura 4: Calificación obtenida por los estudiantes en la propuesta y en la presentación final del proyecto

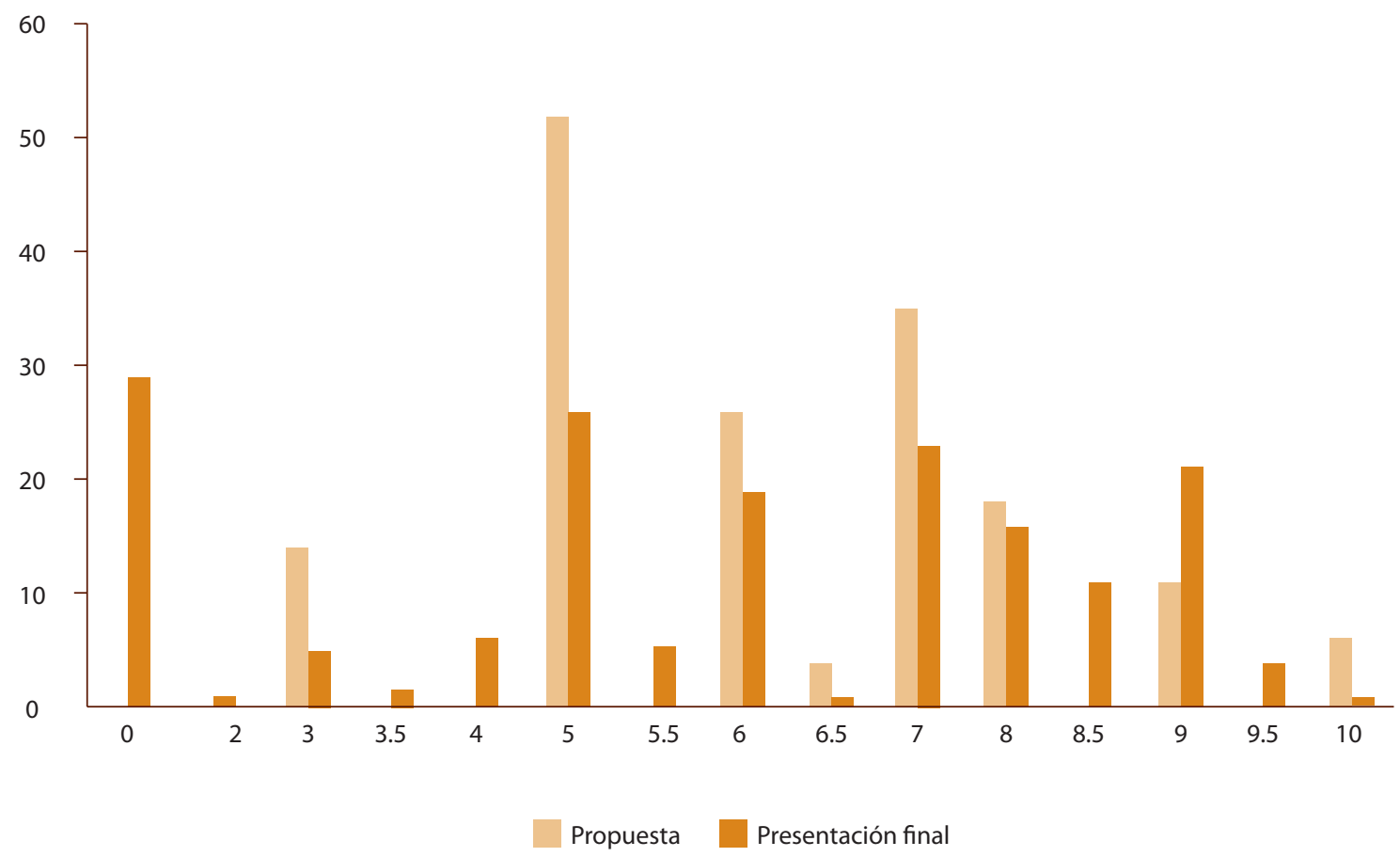

Nota: Elaboración propia.

\section{En relación con el profesorado}

- Formación académica, experiencia en docencia y en estrategias de aprendizaje basados en solución de problemas

La mayoría del profesorado que impartió las materias del proyecto integrador $(77,7 \%)$ posee formación de tercer y cuarto nivel en ingeniería y áreas afines, y cuenta con experiencia en docencia universitaria de cinco o más años. 
http://doi.org/10.15359/ree.25-2.2

En relación con la capacitación del profesorado en estrategias de aprendizaje basadas en solución de problemas mediante proyectos integradores u otras estrategias de aprendizaje similares, el 55,55\% del profesorado declaró haber recibido capacitación sobre estas temáticas y solo el 22,22\% la recibió en la UTM; sin embargo, todo el profesorado participante en este estudio considera necesario recibir capacitación sobre la implementación de PIS. Este aspecto toma especial relevancia en el profesorado de carreras como Ingeniería Química, por no contar con estudios formales en el área de la pedagogía. Esto es consecuente con lo planteado por Solaz-Portolés et al. (2011), quienes indican que al ser las disciplinas de carácter científico las que mejor se adaptan a métodos de aprendizajes basados en resolución de problemas, se hace imprescindible la formación del profesorado en esta metodología.

La mayoría del profesorado (66,66\%) indica no haber aplicado anteriormente estrategias basadas en solución de problemas en sus cursos, como lo son los PIS. Para un porcentaje igual de docentes la experiencia en la aplicación del proyecto integrador como estrategia pedagógica fue favorable, la opinión contraria del resto del profesorado se debe, principalmente, a la falta de interés por parte del estudiantado ante esta metodología de aprendizaje. En esta respuesta también ha debido influir el número de grupos asignados a cada docente, pues la percepción puede ser diferente para quienes tuvieron a cargo solo tres grupos, en relación con docentes a quienes se les designaron cinco o siete grupos.

\section{- Planteamiento de la propuesta}

Durante la primera etapa de los PIS, la mayoría del profesorado declara haber presentado al menos dos dificultades resaltantes durante la realización de las propuestas de los proyectos por parte del estudiantado. La primera fue la dificultad del estudiantado para identificar problemas del entorno que pudieran ser abordados a partir de los PIS, aspecto que también fue resaltado por el estudiantado. Además, algún profesorado determinó una falta total de comprensión y sistematización de las actividades a realizar por parte del estudiantado. En este sentido, otras investigaciones exponen la necesidad de aplicar, particularmente en los primeros años de la carrera, una evaluación diagnóstica para conocer el nivel del estudiantado, así como la diferencias entre este, lo cual permitiría conocer qué medidas de refuerzo se pueden sugerir y, finalmente, qué alcance pueden tener los PIS (Díaz Lantada et al., 2013).

La segunda dificultad expresada por el profesorado fue el tiempo que requieren las actividades asociadas al proyecto, principalmente en las primeras semanas del periodo académico, debido a la dificultad del estudiantado para comprender los objetivos que se plantea alcanzar con el proyecto integrador. De allí que la mayoría del profesorado reconoció que las materias que conforman el proyecto integrador requieren más horas de dedicación por parte del profesorado, que aquellas que no pertenecen a él y que tienen el mismo número de créditos académico u horas de clases. Esto se debe, principalmente, a que en las asignaturas 
http://doi.org/10.15359/ree.25-2.2

http://www.una.ac.cr/educare

educare@una.ac.cr

integradoras, la interacción docente-estudiante es mayor debido a la inherente variabilidad de situaciones que se presentan cuando se está en la búsqueda de resolver problemas, lo que requiere de un tiempo de dedicación adicional que, en muchos casos, no es reconocido por la administración académica de las instituciones de educación (Chinowsky et al., 2006).

Entre las actividades que más tiempo demandan en la primera etapa de los PIS están: organización del curso, preparación del estudiantado, retrasos en los proyectos, cambios de participantes o restricciones de acceso. Esto hace que no sea práctico la aplicación de esta metodología a cursos numerosos (Chinowsky et al., 2006).

\section{- Temática central de los PIS y cátedra integradora}

En relación con la temática central establecida para el proyecto, el profesorado cree conveniente que debería ser más amplia y tener solo como límites los temas que se abordan en las tres asignaturas integradoras; esto, con la finalidad de poder abarcar un mayor número de problemas, y con ello evitar la duplicidad o plagio de proyectos durante el mismo período o en períodos subsiguientes. Ya otras personas autoras han determinado que esta es la mejor manera de eliminar el problema de que los proyectos coincidan o tengan componentes similares o repetidos (Díaz Lantada et al., 2013).

La asignatura establecida como cátedra integradora en el rediseño de la carrera es Química Orgánica y Laboratorio l; sin embargo, el 66,7\% del profesorado indica que la asignatura integradora del segundo nivel debería ser Introducción a la Ingeniería Química, por ser la materia que más aporta al perfil de egreso de ingeniería química, aspecto fundamental al momento de seleccionar una asignatura como cátedra integradora.

\section{- Número de integrantes por grupo y por curso}

En cuanto al número de integrantes por grupo, al menos el 44,44\% del profesorado indicó que los grupos deberían ser más reducidos, con la finalidad de garantizar que todo el estudiantado tuviese una participación significativa y más activa en la realización del proyecto, y para evitar que solo uno o dos estudiantes realicen las actividades asociadas al proyecto. Aspecto que también fue resaltado por el estudiantado. Cabe destacar que, reducir el número de participantes por grupo, llevaría a la conformación de más grupos, lo que representaría mayor trabajo de tutoría para el profesorado; por lo que se infiere que esa sea la razón por la cual la mayoría del profesorado indica que seis estudiantes por grupo era adecuado.

En estudios previos se ha determinado que un elevado número de estudiantes por grupo dificulta la interacción docente-estudiante, y con ello la obtención de mejores resultados (Villalobos Delgado et al., 2016). Otros estudios recomiendan que cuando se trabaja con cursos 
http://doi.org/10.15359/ree.25-2.2

numerosos, los grupos deben estar conformados por tres o cuatro estudiantes, pues grupos más grandes pueden fomentar la aparición de miembros parásitos, además de dificultar el componente evaluativo individual en los grupos (Díaz Lantada et al., 2013).

Otro aspecto importante en la implementación de esta metodología de aprendizaje es el número de estudiantes por curso, pues se ha determinado que grupos reducidos de estudiantes ha favorecido la implementación de este tipo de metodologías de aprendizaje (Fernández y Aguado, 2017). Como se pudo apreciar en la Figura 1, el número por curso varió desde 20 estudiantes en el caso del curso $C$ de la asignatura Introducción a la Ingeniería Química, hasta cursos con 50 estudiantes como fue el caso del curso A de la asignatura Química Orgánica y Laboratorio I. En este sentido, se debe trabajar en dos aspectos: a) unificar el número de estudiantes por curso en las diferentes asignaturas, y b) que a su vez este número no exceda los 30 estudiantes por curso.

\section{- Asistencia a tutorías}

El acompañamiento académico a través de tutorías es fundamental en este tipo de estrategias de aprendizaje, ya que ayudan a facilitar los procesos que se desarrollan para abordar el problema. Su papel incluye, entre otros aspectos, promover el trabajo en el grupo y encausarlo "hacia los objetivos propuestos, orientar en el uso de las fuentes de información, y establecer un buen clima de trabajo entre estudiantes en el grupo" (Solaz-Portolés et al., 2011 , p. 179). Las tutorías permiten alcanzar los objetivos que se plantean en los PIS, pues sin el acompañamiento del profesorado-tutor es poco probable que el estudiantado pueda realizar un proyecto de investigación que se adapte a los requerimientos establecidos, debido a la falta de preparación y experiencia que el estudiantado del segundo nivel de Ingeniería Química pudiera tener para desarrollar de forma autónoma un proyecto de investigación. En relación con esto, solo al 22,22\% del profesorado le asistieron todos los grupos a su cargo a tutorías, lo que hace necesario superar las dificultades planteadas previamente por el estudiantado, para que los grupos de trabajo reciban el acompañamiento académico que se requiere al desarrollar proyectos integradores.

\section{- Ponderación de la calificación de los proyectos en la calificación de las asignaturas}

La suma de la calificación asignada por el profesorado tutor en las dos etapas del proyecto representa el $20 \%$ de la calificación total de las tres asignaturas integradoras. La calificación que recibe el estudiantado sobre la presentación de los resultados obtenidos en el proyecto y que se hace al final del periodo académico representa solo el $10 \%$ de la nota final de cada asignatura. Previo a la presentación final del proyecto, el estudiantado pudiera aprobar las asignaturas integradoras, si al sumar las calificaciones obtenidas en todos los componentes de evaluación 
http://doi.org/10.15359/ree.25-2.2

http://www.una.ac.cr/educare

educare@una.ac.cr

alcanza al menos el 70\% de la calificación máxima (70 puntos). El hecho de que la presentación final del proyecto integrador no es obligatoria para la aprobación de las asignaturas integradoras ha llevado a algún estudiantado a desistir de la presentación del PIS, debido a que la calificación que obtendrían no sería necesaria para aprobar las asignaturas, evitando con ello tanto el estrés como el trabajo asociado a la realización de una presentación escrita y oral.

En este estudio, dos de los 33 grupos de trabajo no realizaron la presentación final del proyecto y, aun así, varios y varias integrantes de estos dos grupos aprobaron las asignaturas del proyecto integrador. Esta situación presenta un agravante adicional, cuando la decisión de no presentar el proyecto final es tomada por el estudiantado que ha asumido el papel del líder del grupo; lo que hace, a su vez, que el resto del grupo también desista de la presentación final del proyecto. Se genera, con esto, un problema al resto del estudiantado del grupo, para quienes sí pudiera ser necesaria la calificación final de proyecto para aprobar las asignaturas integradoras. Con la finalidad de evitar situaciones como la anteriormente expuesta, se debe considerar establecer como obligatorio la realización y presentación de los PIS, para la aprobación de las asignaturas integradoras.

\section{Conclusiones}

La implementación de una estrategia de aprendizaje no debe responder solo a resultados favorables en su aplicación previa, pues esto, por sí solo, no garantiza que se obtendrán los mismos resultados. En el caso de los PIS, previo a su implementación, se deben considerar muchos aspectos de índole organizacional asociados a los tres principales actores (institución, docentes y estudiantes) $y$, de esta manera, lograr que esta sea una experiencia de aprendizaje favorable tanto para el estudiantado como para el profesorado.

En el caso particular de la UTM, se hace necesario establecer normas para la implementación de los PIS, en las que se consideren aspectos como: inscripción de estudiantes en los cursos de las asignaturas del proyecto integrador por grupos de trabajo, unificación del número máximo de estudiantes por curso y de estudiantes por grupo, reconocimiento de carga horaria adicional para docentes que impartan asignaturas de proyectos integradores, obligatoriedad de la realización y presentación de los PIS por parte del estudiantado para la aprobación de las asignaturas, entre otros.

Es necesario que el profesorado que imparte asignaturas pertenecientes a los proyectos integradores reciba capacitación docente previa en estas estrategias de aprendizaje basadas en solución de problemas. En el caso del estudiantado, este debe conocer la metodología de trabajo de los PIS, poseer formación en técnicas de estudio y trabajo en equipo, y tener conocimientos previos sobre la temática a abordar en el proyecto integrador; aspectos fundamentales para lograr la consecución de los PIS, y que pueden ser evaluados mediante la implementación de pruebas diagnósticas. 
http://doi.org/10.15359/ree.25-2.2

\section{Recomendaciones}

A continuación, se presentan algunas recomendaciones de índole práctico que permitirían, con su implementación, desarrollar en el estudiantado competencias personales y profesionales asociadas a la realización de PIS. Estas recomendaciones son:

- Reconsiderar la realización de PIS en los primeros niveles de la carrera.

- Establecer obligatoriedad en la realización y presentación final de los PIS.

- Realizar la inscripción del estudiantado en los cursos de las asignaturas integradoras, en grupos de trabajo.

- Establecer un número de estudiantes por curso y un número de estudiantes por grupo que se ajuste a estrategias de aprendizajes basadas en solución problemas.

- Establecer programas de capacitación tanto para el estudiantado, como para el profesorado, en estrategias de aprendizaje basadas en resolución de problemas mediante trabajo cooperativo.

- Establecer horarios exclusivos solo para tutorías, por cada nivel de la carrera, en lo que no se impartan otras asignaturas.

- Limitar la temática a abordar en los PIS solo al contenido de las asignaturas integradoras.

- Reconocer al profesorado el tiempo adicional que requiere la impartición de asignaturas perteneciente a PIS.

\section{Referencias}

Castillejo-Olán, R., Rodríguez-Torres, Á. F., Páez-Granja, R. E., Altamirano-Vaca, E. J. y GranadosRomero, J. F. (2017). El proyecto integrador de saberes. Análisis crítico desde la perspectiva de alumnos y docentes (revisión). Olimpia: Revista de la facultad de cultura física de la Universidad de Granma, 14(46), 99-110. https://dialnet.unirioja.es/servlet/ articulo?codigo $=6220153$

Chinowsky, P. S., Brown, H., Szajnman, A., \& Realph, A. (2006). Developing knowledge landscapes through project-based learning. Journal of Professional Issues in Engineering Education and Practice, 132(2), 118-124. https://doi.org/10.1061/(ASCE)1052-3928(2006)132:2(118)

de los Ríos, I., Cazorla, A., Díaz-Puente, J. M. y Yagüe, J. L. (2010). Project-based learning in engineering higher education:Two decades of teaching competences in real environments. Procedia - Social and Behavioral Sciences, 2(2), 1368-1378. https://doi.org/10.1016/j. sbspro.2010.03.202 
http://doi.org/10.15359/ree.25-2.2

http://www.una.ac.cr/educare

educare@una.ac.cr

Díaz Lantada, A., Lafont Morgado, P., Muñoz-Guijosa, J. M., Muñoz Sanz, J. L., Echávarri Otero, J., Muñoz García, J., Chacón Tanarro, E. y de la Guerra Ochoa, E. (2013). Towards successful project-based teaching-learning experiences in engineering education. International Journal of Engineering Education, 29(2), 1-15.

Egido Gálvez, I., Aranda Redruello, R., Cerrillo Martín, R., de la Herrán Gascón, A., de Miguel Badesa, S., Gómez García, M., Hernández Castilla, R., Izuzquiza Gasset, D., Murillo Torrecilla, F. J., Pérez Serrano, M. y Rodríguez, R. M. (2007). El aprendizaje basado en problemas como innovación docente en la universidad: Posibilidades y limitaciones. Educación y Futuro, 16, 85-100. https://cesdonbosco.com/documentos/revistaeyf/EYF 16.pdf

Fernández, C. L. y Aguado, M. I. (2017). Aprendizaje basado en problemas como complemento de la enseñanza tradicional en fisicoquímica. Educación Química, 28(3), 154-162. https:// doi.org/10.1016/j.eq.2017.03.001

Fioravanti, M. L., Sena, B., Paschoal, L. N., Silva, L. R., Allian, A. P., Nakagawa, E. Y., Souza, S. R. S., Isotani, S. y Barbosa, E. F. (2018). Integrating project based learning and project management for software engineering teaching: An experience report. En Proceedings of the 49th ACM Technical Symposium on Computer Science Education (pp. 806-811). ACM. https://doi.org/10.1145/3159450.3159599

Hewitt Ramírez, N. (2007). El proyecto integrador: Una estrategia pedagógica para lograr la integración y la socialización del conocimiento. Psychologia. Avances de la disciplina., 1(1), 235-240. https://www.redalyc.org/articulo.oa?id=297224869006

Johnson, D. W., Johnson, R. T. y Holubec, E. J. (1999). El aprendizaje cooperativo en el aula. Paidós.

Lanza Escobedo, D. y Barrios Fernández, Á. (2012). Aprendizaje cooperativo como fórmula para el desarrollo de competencias en el espacio europeo de educación superior: Un estudio exploratorio con alumnos de psicología de la Universidad Autónoma de Madrid. CIDU, 1, 1-20. https://www.cidui.org/revistacidui/index.php/cidui/article/view/123

Moscoso Zamora, V. H. y Quiñonez Alvarado, E. S. (2018). Proyecto integrador de saberes, evidencia del resultado de aprendizaje. INNOVA Research Journal, 3(3), 84-94. https://doi. org/10.33890/innova.v3.n3.2018.444

Organización para la Cooperación y el Desarrollo Económico. (2007). PISA 2006. Marco de la evaluación. Conocimientos y habilidades en ciencias, matemáticas y lectura. Santillana. https://doi.org/10.1787/9789264066168-es

Savage, R. N., Chen, K. C. y Vanasupa, L. (2009). Integrating project-based learning throughout the undergraduate engineering curriculum. IEEE Engineering Management Review, 37(1), 1-18. https://doi.org/10.1109/EMR.2009.4804346 
http://doi.org/10.15359/ree.25-2.2

http://www.una.ac.cr/educare educare@una.ac.cr

Solaz-Portolés, J. J., López, V. S. y Gómez López, Á. G. (2011). Aprendizaje basado en problemas en la educación superior: Una metodología necesaria en la formación del profesorado. Didáctica de las ciencias experimentales y sociales, 25, 177-186. Recuperado de https:// dialnet.unirioja.es/servlet/articulo?codigo $=3766344$

Universidad Técnica de Manabí. (2016). Rediseño de la carrera de Ingeniería Química. Autor.

Villalobos Delgado, V., Ávila Palet, J. E., Olivares, S. L. (2016). Aprendizaje basado en problemas en química y el pensamiento crítico en secundaria. Revista Mexicana de Investigación Educativa, 21(69), 557-581. http://www.scielo.org.mx/scielo.php?script=sci arttext\&pid $=\mathrm{S} 1405-66662016000200557$ 